\title{
Deep neural networks for COVID-19 detection and diagnosis using images and acoustic-based techniques: a recent review
}

\author{
Walid Hariri ${ }^{1}$ (D) Ali Narin ${ }^{2}$
}

Accepted: 7 August 2021 / Published online: 24 August 2021

(c) The Author(s), under exclusive licence to Springer-Verlag GmbH Germany, part of Springer Nature 2021

\begin{abstract}
The new coronavirus disease (COVID-19) has been declared a pandemic since March 2020 by the World Health Organization. It consists of an emerging viral infection with respiratory tropism that could develop atypical pneumonia. Experts emphasize the importance of early detection of those who have the COVID-19 virus. In this way, patients will be isolated from other people and the spread of the virus can be prevented. For this reason, it has become an area of interest to develop early diagnosis and detection methods to ensure a rapid treatment process and prevent the virus from spreading. Since the standard testing system is time-consuming and not available for everyone, alternative early screening techniques have become an urgent need. In this study, the approaches used in the detection of COVID-19 based on deep learning (DL) algorithms, which have been popular in recent years, have been comprehensively discussed. The advantages and disadvantages of different approaches used in literature are examined in detail. We further present the databases and major future challenges of DL-based COVID-19 detection. The computed tomography of the chest and X-ray images gives a rich representation of the patient's lung that is less time-consuming and allows an efficient viral pneumonia detection using the DL algorithms. The first step is the preprocessing of these images to remove noise. Next, deep features are extracted using multiple types of deep models (pretrained models, generative models, generic neural networks, etc.). Finally, the classification is performed using the obtained features to decide whether the patient is infected by coronavirus or it is another lung disease. In this study, we also give a brief review of the latest applications of cough analysis to early screen the COVID-19 and human mobility estimation to limit its spread.
\end{abstract}

Keywords Viral pneumonia $\cdot$ COVID-19 $\cdot$ Deep learning $\cdot$ Chest CT scan $\cdot$ X-ray image $\cdot$ Cough analysis

\section{Introduction}

The novel severe acute respiratory syndrome-related coronavirus (SARS-CoV-2) started from Wuhan, China, in December 2019 and spread to all the countries worldwide. This virus caused pneumonia of unknown cytology and is named COVID-19. This infectious disease has been classified as a public health crisis of the international community concern on January 30, 2020, because of its high infectivity and mortality. In March 28, 2020, 177 countries have been the most

Walid Hariri

hariri@labged.net

Ali Narin

alinarin@beun.edu.tr

1 Labged Laboratory, Computer Science Department, Badji Mokhtar Annaba University, Annaba, Algeria

2 Department of Electrical and Electronics Engineering, Zonguldak Bulent Ecevit University, Zonguldak, Turkey impacted countries worldwide with more than 600,000 cases. Italy was the second country after China with 92,472 cases. Detailed statistics about the spread of the virus in Italy can be found in Abenavoli et al. (2020).

In 2021, many variants of COVID-19 have been detected in different countries including UK, Brazil and India. The spread of these variants and the mortality rates are more important than the previous ones. Figure 1 presents the daily cases of the Indian variant reported by the COVID-19 study group in India. The lack of successful diagnosis or preventive measures has led to a rise in the number of cases, an increase in the cost of hospitalizations and palliative treatments. Therefore, scientists and medical industries around the world incited to find a prompt and accurate detection of COVID-19 for early prevention, screening, forecasting, drug development and contact tracing to save more time for the scientific community and healthcare expert to pass to the next diagnosis stage to reduce the death rate reverse transcription polymerase chain reaction (RT-PCR) is rec- 


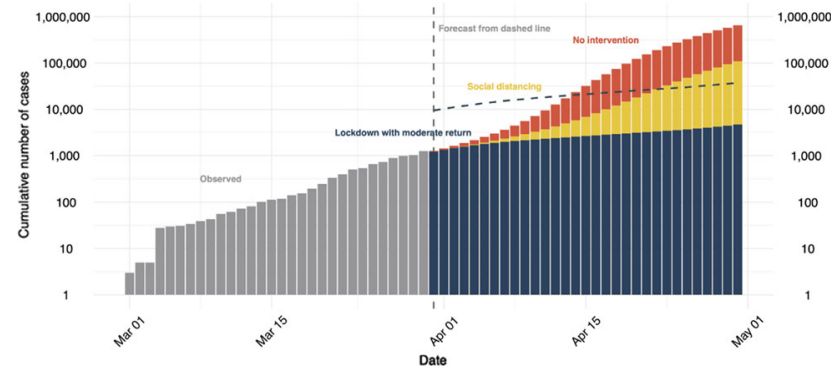

Fig. 1 Cumulative daily cases in India Group (2021)

ommended to diagnose COVID-19. Additionally, there are studies in the literature using various imaging methods (computed tomography (CT) and X-ray). (Xu et al. 2020; Gozes et al. 2020; Ucar and Korkmaz 2020; Sethy and Behera 2020; Zhang et al. 2020a). It may occur in situations that negatively affect these methods. The changes of viruses by the appearance of new mutations make the classifications a more challenging task (Grubaugh et al. 2020). Moreover, one of the biggest problems with COVID-19 patients is viral pneumonia (VP). Differentiating between viral and non-viral pneumonia (nVP) is not easy. Coexistence of COVID-19 and viral pneumonia can have dire consequences.

Oxford COVID-19 Evidence Service Team Center follows some tips in identifying these problems. Muscle pain, loss of sense of smell and shortness of breath without pleuritic pain are the most common symptoms, especially in the case of COVID-19 infection. On the other hand, symptoms such as bilateral positive lung findings, tachycardia or tachypnea disproportionate to temperature and low temperature indicate other VP symptoms (Heneghan 2020). nVP, however, is most susceptible if it becomes rapidly unwell after a short period from the appearance of symptoms and does not have similar symptoms of COVID-19, pleuritic pain or purulent sputum.

Many studies have been introduced to solve this problem, for instance, Zhang et al. (2020b) proposed to lessen the process of anomaly detection into a one-class-classification problem using a confidence aware module. Deep learning (DL) is then used for the classification task as shown in Fig. 2. Recent reviews show that the use of novel technology with artificial intelligence (AI) and machine learning (ML) techniques considerably improves the detection, screening, contact tracing, forecasting and vaccine advance with high reliability.

Since the COVID-19 pandemic started, it has been clear that deep learning algorithms from ML technologies seem to be used extensively to detect COVID-19, VP, bacterial pneumonia (BP) and other similar cases. The advantages and disadvantages of these studies should be evaluated. In this study, it is aimed to present a detailed review on studies using DL approaches using various images in the literature to detect COVID-19. In addition, studies that detection of COVID-19 using acoustic sound data are included.

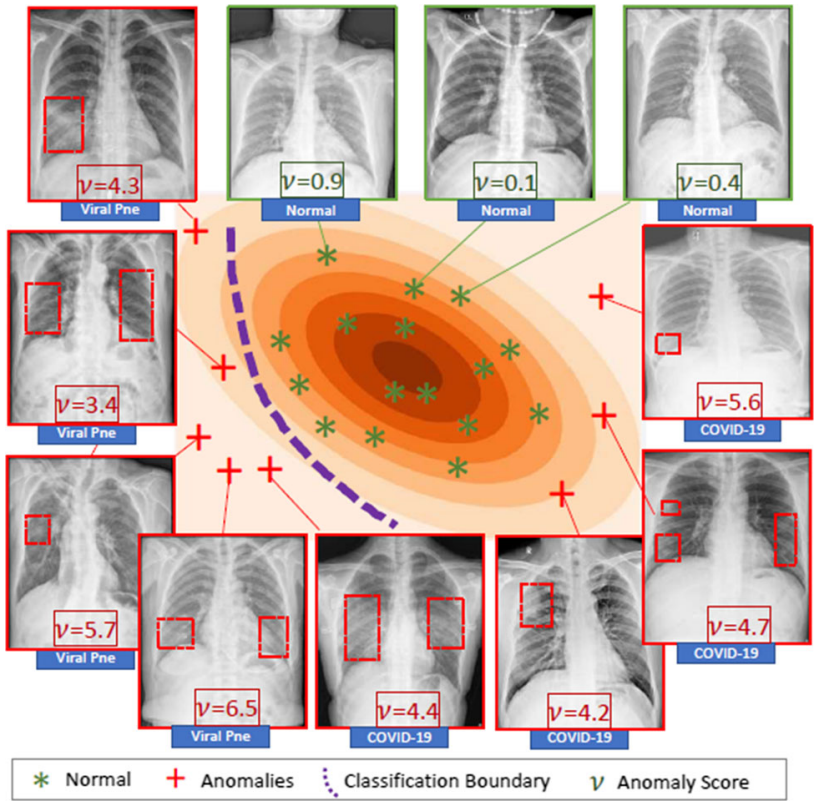

Fig. 2 Distinguishing between VP cases (anomalies) and nVP cases and normal controls (Zhang et al. 2020b)

In this review paper, known databases, involving IEEE Xplore, NCBI, ScienceDirect, SpringerLink, ACM Digital Library and ArXiv, have been used to seek for coronavirus related research. Most papers are chosen from journals, conference papers and preprints using Google Scholar. Relevant X-ray and CT scan databases for COVID-19 detection have been mainly found on the Kaggle website. The articles are selected using the keywords COVID-19, Cough, Radiography Database, Coronavirus, Deep Learning, Transfer learning, pre-trained models, Detection, Diagnosis and Segmentation. The last update of the papers research using the aforementioned keywords has been made on July 30, 2021.

The rest of this paper is organized as follows: Sect. 2 presents the medical imaging technologies. In Sect. 3 we review the most important DL methods proposed to diagnose the COVID-19, as well as the recent advanced applications. Section 4 presents some additional DL applications to fight against COVID-19 such as acoustic analysis and human mobility estimation. In Sect. 5 an overall discussion and proposed solutions are presented to accurately diagnose and to reduce the spread of the COVID-19. Conclusions, future trends and challenges end the paper.

\section{Medical imaging technologies versus RT-PCR}

The medical imaging field has considerably emerged in the last years offering reliable automated methods for clinical 
decision making. It has received wide acceptance by the scientists and the medical community. In the case of COVID19, CT scans and X-ray images can play a vital role in the early diagnosis of the disease. Infected patients have clinical symptoms including cough and fever; however, an important proportion of infected patients can be asymptomatic. In Germany, it has been confirmed in the study of Rothe et al. (2020) that an asymptomatic patient was able to transmit the virus to another patient. According to the study of Al-Tawfiq (2020) from 9 countries, 18 from 144 cases were asymptomatic, the equivalent of $12.5 \%$. The study has been done using the RT-PCR test.

Due to the high risk of transmission of COVID-19, accurate diagnostic methods are urgently needed to prevent the spread of the virus and for humanity to breathe comfortably. Besides being the gold standard of the RT-PCR test, the results are time-consuming (requires 5-6 h) to obtain. In addition, the high rate of false detection of RT-PCR test is questioned whether it is a good diagnostic method. Xie et al. (2020), Long et al. (2020). In this case, it is recommended that patients with typical imaging findings should be separated ones from one another and more than one RT-PCR test should performed to avoid misdiagnosis.

The X-ray, however, is an efficient screening method; it is fast at capturing, cheaper than the RT-PCR test and largely available worldwide. CT scans, on the other hand, can be obtained much faster and more accurately in the presence of an efficient algorithm (notably DL algorithms) to accurately identify the infected patients. In Liu et al. (2019), it has been proven that DL offers highly promising results for medical diagnostics compared to healthcare professionals. Figure 3 presents the change that occurs in the COVID-19 pneumonia cases on some days. In the following section, detailed information about CT and X-ray images is presented.

\subsection{Chest computed tomography}

$\mathrm{CT}$ is an imaging method that uses a special X-ray beam to create detailed scans of areas inside the body (e.g., lungs, heart, blood vessels, airways and lymph nodes). These images are taken from different angles to generate tomographic images which give the possibility to the radiographers to directly see inside the body instead of surgery. CT images are an effective way of making clinical decisions. They showed high efficiency in diagnosing COVID-19 especially patients with false-negative RT-PCR results, assuming a role for the CT as a reliable tool for COVID-19 diagnosis during this pandemic ( $\mathrm{Li}$ and $\mathrm{Xia}$ 2020; Ai et al. 2020; Xie et al. 2020; Huang et al. 2020). Therefore, the National Health Commission of the People's Republic of China suggested CT examination in monitoring disease progression and controlling treatments of COVID-19 in its $6^{\text {th }}$ version of the diagnosis and treatment program (Zhao et al. 2020).

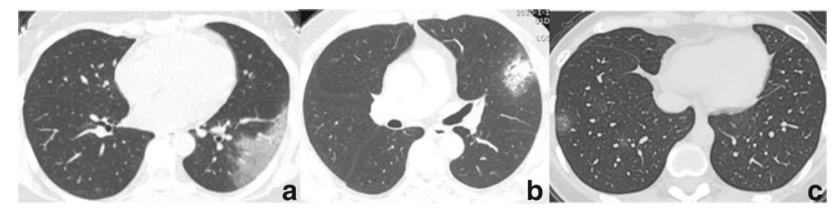

Fig. 3 CT scans in the early fast gradually stage of COVID-19 pneumonia cases. a GGO plus reticular pattern on the forth day. b GGO plus consolidation on the third day. $\mathbf{c}$ GGO on the second day (Zhou et al. 2020)

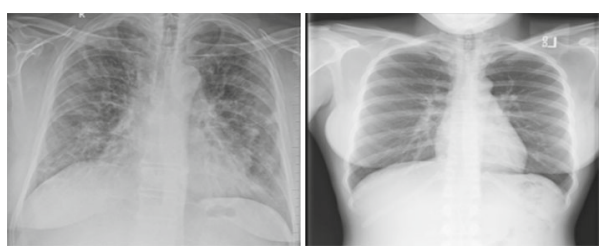

Fig. 4 An example of X-ray images for a COVID-19 patient (left) and normal case (right)

\subsection{X-ray image}

Wilhelm Conrad Röntgen has discovered the first X-ray in 1895 during experimenting with Lenard tubes and Crookes tubes. X-ray has a very important role in the medical field, it can help in the prevention of infection, diagnosis and control. $\mathrm{X}$-ray scans are used worldwide to diagnose the injured part and for the detection or other diseases in order to treat patients (Ghosh and Saha 2018). The X-ray facility is available even in the remotest parts, and thus, X-ray images can be easily acquired for patients even in their home or in their quarantine location. These images have been extensively used for COVID-19 diagnosis (Narin et al. 2020). The most common reported abnormal in chest X-ray (CXR) findings are groundglass opacities (GGOs) (Yoon et al. 2020). Figure 4 presents an example of an X-ray scans for COVID-19 and normal cases. CXR is the most widely used imaging technology by researchers because it is easily available and inexpensive.

\section{Deep learning approaches in the COVID-19 pandemic}

DL is a subset of ML that offers considerable power for improving the accuracy and speed of diagnosis by automating the screening through medical imaging in collaboration with radiologists and/or physicians. Subsequently, it has received wide acceptance and interest by the medical community leads to emphasizing the development of such diagnostic technologies (Liu et al. 2019). For example, Cinaglia et al. (2018) have presented initial results on a framework for the acquisition and decomposition of DICOM images. The tests have been conducted on a dataset from University Hospital of Catanzaro for segmentation and anatomical features extraction from 
Fig. 5 Taxonomy of deep learning-based approaches for COVID-19 diagnosis
Deep Learning Approaches for COVID-19 Diagnosis

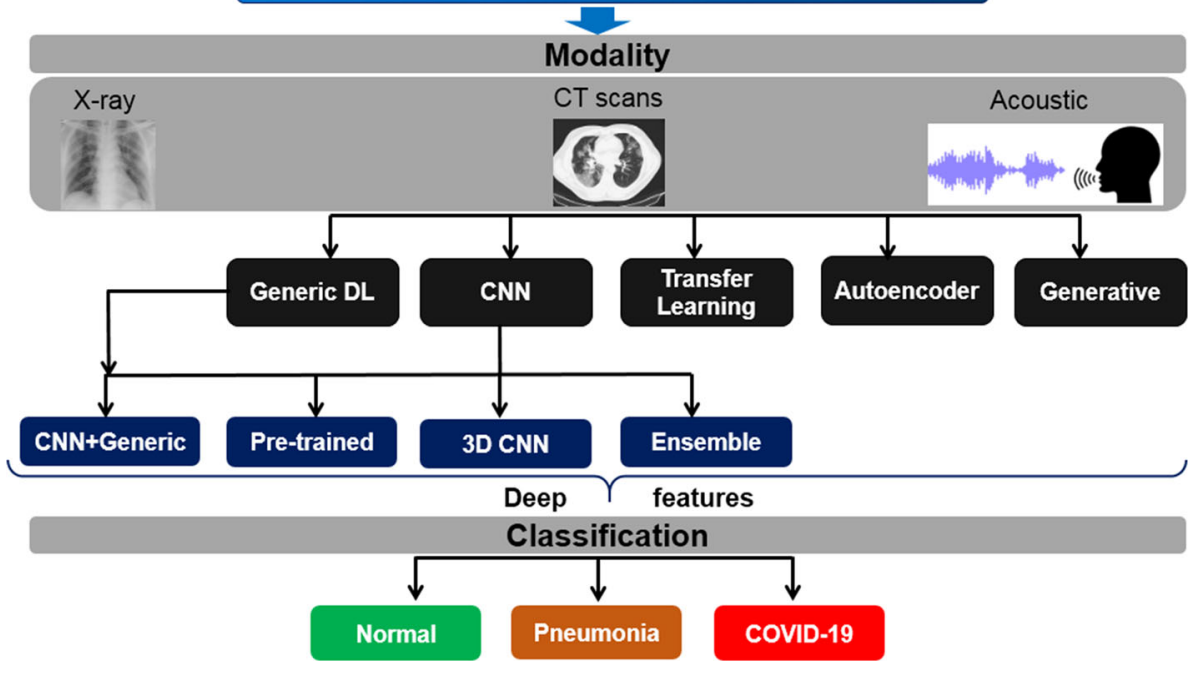

lungs. Information entropy is another image analysis technique in cryptographic applications; it has been applied in the context of COVID-19 as presented in Javan et al. (2021).

In the following, we review the most important DL approaches adopted to diagnose the pneumonia of COVID19 since its spread in December 2019 until today. Figure 5 presents the taxonomy of these approaches using different images and acoustic features. In the following, we detail each of the distinguished nine groups:

\subsection{Generic deep learning}

Generic DL methods without any specific modification have been proposed to detect COVID-19. For example, Wang et al. (2020d) have used CT images of 5372 patients from 7 different cities in China to train a deep neural network (DNN).

Pneumonia Detection Challenge dataset (RSNA) is used in Luz et al. (2020) to train a DL model in order to locate lung opacities on chest radiographs. RSNA dataset contains two classes: normal and pneumonia (non-normal). The total of 16,680 images have been used from this data set where 8066 are from healthy class (normal), whereas 8614 are classified as pneumonia.

The authors in Song et al. (2020) collected from two hospitals of in China the CT images of 88 infected patients (COVID-19) and 101 patients diagnosed with bacteria pneumonia, where the rest are healthy (86 persons). Using this dataset, they applied a DL-based CT diagnosis system namely: DeepPneumonia to localize the principal lesion features, especially GGO and thus to identify the infected patients. The first step is the segmentation of the lung region. Next, they introduced the DRE-Net (detailed relation extraction neural network) to draw the top-K features in the CT images and to receive the image-level predictions. Finally, the image-level predictions are used to diagnose the patient.
Another generic DL framework is proposed in Zhang et al. (2020) to automatically extract and analyze regions with high possibility to be infected with COVID-19. To do so, the authors applied a segmentation stage using a DL-based technique. Then, the infected regions were processed and quantized using specific metrics in the CT scan.

We can also find generic convolutional neural networks in which the authors use the generic CNN trained with their datasets without any combination with other ML algorithms or pretrained models. For example, in Fu et al. (2020), the authors trained the CNN model with the data collected from Wuhan Jin Yin-Tan hospital in order to classify the CT images into one of the five following classes: healthy lung, COVID-19, pneumonia, non-COVID-19 VP, BP and pulmonary tuberculosis.

\subsection{Transfer learning}

Transfer learning (TL) is a ML technique in which a trained model for one task is redesigned in a related second task (see Fig. 6). This approach is explicitly useful when there are not sufficient datasets like in the case of COVID-19 in order to either reduce the necessary fine-tuning data size or improve performance. TL can be used in two scenarios: supervised (with labeled data from the target domain) or unsupervised (without any labeled data from the target domain: the pretraining process is supervised, but unsupervised during fine-tuning). A DNN is proposed in Jaiswal et al. (2020) to detect COVID-19 using X-ray images. To do so, the authors applied a TL approach on the deep Pruned EfficientNet model. Then, it has been interpolated by post-hoc analysis to be able to explain the obtained predictions. TL based-framework for the detection of pneumonia is proposed in Chouhan et al. (2020). The features have been extracted from X-ray images using five different pretrained models: 


\subsection{Data augmentation and generation techniques}

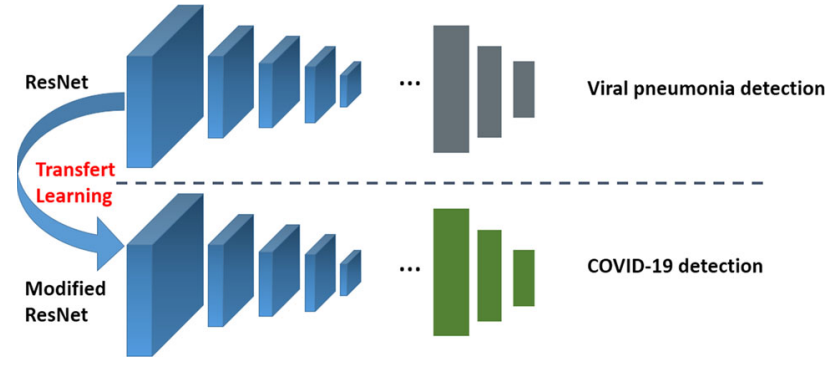

Fig. 6 An example of transfer learning process for COVID-19 detection

DenseNet121, ResNet18, GoogLeNet, AlexNet and InceptionV3. Next, an ensemble model has been added to combine outputs from all pretrained models. The obtained results are as follows: accuracy of $96.4 \%$ and recall of $99.62 \%$ on nontrained data from the Guangzhou Women and Children's Medical Center database.

Fine-tuned deep TL with generative adversarial network (GAN) is presented in Khalifa et al. (2020) to learn a limited dataset and to avoid the overfitting problem. To do so, the authors applied the pretrained models: Squeeznet, AlexNet, GoogLeNet and Resnet18 as deep TL models to detect pneumonia from chest X-rays. Applying a combination of GAN and deep transfer models enhanced the accuracy of the proposed system and realized 99\%. After applying image preprocessing algorithms to the chest X-ray images to identify and remove diaphragm regions, the pretrained VGG-16 model (Simonyan and Zisserman 2014) has been fine-tuned in Heidari et al. (2020) using the obtained images to predict COVID-19 infected pneumonia. Another work proposed in Apostolopoulos and Mpesiana (2020) to detect the COVID19 in small medical image datasets. To do so, they worked with two different data sets from public databases. In the first dataset, there are 224 COVID-19, 700 BP and 504 normal $\mathrm{X}$-ray images. The second dataset includes 224 COVID-19, $714 \mathrm{BP}$ and VP and 504 normal X-ray images. They obtained $96.78 \%$ accuracy, $98.66 \%$ sensitivity and $96.46 \%$ specificity performance values.

Multi-channel TL-based method with X-ray images have been proposed in Misra et al. (2020). Multi-channel pretrained ResNet model is then used to perform the diagnosis of COVID-19. To classify the X-ray images on a one-againstall strategy, three ResNet models have been retrained. The three allowed classifications are: (1) normal or diseased, (2) pneumonia or non-pneumonia and (3) COVID-19 or nonCOVID-19 individuals. The method achieved a precision of $94 \%$ and a recall of $100 \%$. Other TL-based methods can be found in (Minaee et al. 2020; Maghdid et al. 2020; Benbrahim et al. 2020; Haghanifar et al. 2020; Abbas et al. 2020; Rahaman et al. 2020; Perumal et al. 2020; Loey et al. 2021).
Recently, generative adversarial networks (GANs) are considered the most powerful and successful method for data augmentation. Since the outbreak of COVID-19 is recent, it is difficult to gather a significant amount of radiographic images and datasets in such a short time. Therefore, DL networks, especially CNNs, need additional training data to overcome this problem and to enhance the efficiency of CNN in detecting COVID-19 (see Fig. 7). Various methods have been applied the GANs for this reason. For instance, in Waheed et al. (2020), authors generate more X-ray images using Auxiliary Classifier Generative Adversarial Network (ACGAN) based on the CovidGAN model. Accordingly, the classification accuracy has been significantly enhanced from $85 \%$ using the CNN alone, to $95 \%$ using the ACGAN with CovidGAN model.

Also, to handle the problem of the lack of datasets for COVID-19, Loey et al. (2020) proposed classical data augmentation techniques along with Conditional GAN (CGAN) on the basis of a deep transfer learning model for COVID-19 detection using CT images. Similar representation has been used in Loey et al. (2020) to classify the CT images into the following four classes : the COVID-19, normal, pneumonia bacterial and pneumonia virus. To do so, the authors have used a dataset of 307 images. Three deep transfer models are then carried out in this work for investigation. The models are the GoogLeNet, AlexNet and ResNet18. Three strategies have been conducted; in each strategy, the authors applied a different deep TL using the three pretrained models mentioned above. The testing accuracies achieved by the GoogLeNet, AlexNet and ResNet18 are 80.6\%, 85.2\% and $100 \%$, respectively.

Another method proposed in Karakanis and Leontidis (2020) aims to generate synthetic medical images using DL CGAN to overcome the dataset limitation that leads to overfitting. The proposed model has been implemented in a form to support a lightweight architecture without transfer learning without performance degradation. It can deal with any non-uniformity in the data distribution and the limited accessibility of training images in the classes. It consists of a single convolutional layer with filter size 32 and kernel 4 $\times 4$, followed by ReLU activation function and Max Pooling layer for down-sampling the image (input representation) and enabling feature extraction. After a flatten layer there exists a dense layer of size 128 , followed by dropout and a final dense layer with softmax activation function for a binary output.

GANs can also be used during the training step along with pretrained models. For example, authors in Ghassemi et al. (2021) have proposed to use CycleGAN for unpaired imageto-image translation. It consists of training two generator discriminators concurrently. The following pretrained models have been employed: ResNet, EfficientNet, Densenet, ViT 


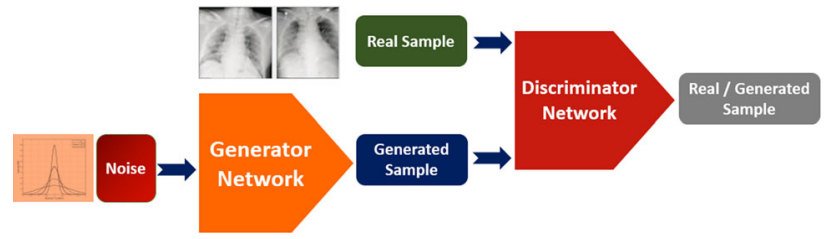

Fig. 7 Generative adversarial network representation for COVID-19 detection

and ResNest. To generate data, the first generator takes the first part of images as input and generates images for the second part. Next, the second generator applies the inverse. The discriminator models will then separate the generated images from origin ones. Finally, it feeds the gradients to the generators. This study has proven that CycleGAN could be successfully used for COVID-19 diagnosis by data augmentation to enhance the accuracy of the pretrained CNNs.

Since supervised learning-based techniques is a very difficult task because of the scarcity of large amount of labeled data, GANs can be used as a semi-supervised learning method to solve this problem for COVID-19 detection. For example, Alizadehsani et al. (2021) used GAN discriminator output for classification. Learning from a merged small amount of labeled data and unlabeled data is carried out. This semi-supervised method gives better performance compared to supervised learning of CNNs. Other methods based on data augmentation to detect the COVID-19 can be found in Ahmed et al. (2021).

\subsection{Autoencoder-based models}

Another ML technique to handle the problem of insufficient data for the affected COVID-19 cases is the autoencoder (AE) (Baldi 2012). It is a deep learning method that contains two effective data encoder and decoder employed for unsupervised feature learning. The $\mathrm{AE}$ models are composed of 2 main steps: encoding and decoding. The entries of this model are mapped to a reduced space dimension while ensuring an accurate feature representation within the encoder. In the second step, the decoding consists of reverting samples to their initial space by generating data from the reduced space representation. Figure 8 shows the structure of autoencoder and its two main steps. The advantage of adopting such unsupervised classification to handle the problem of COVID-19 detection compared to its counterpart (supervised classification) is to avoid the long time spent in assembling large amounts of data which could increase the risk of mortality and postpones medical care. For example, in Khoshbakhtian et al. (2020), the authors introduced the COVIDomaly which aims to diagnose new COVID-19 cases using a convolutional autoencoder framework. They tested two strategies on the COVIDX dataset acquired from the chest radiographs

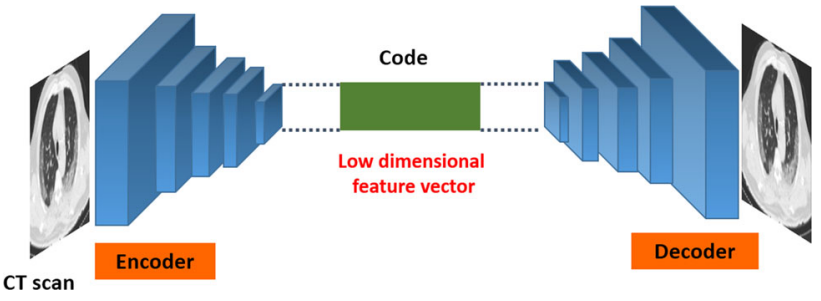

Fig. 8 An example of autoencoder model for COVID-19 detection

by training the model on chest X-rays: the first strategy used only healthy adults, the second tested healthy and BP, and infected adults with COVID-19. Using 3-fold crossvalidation, they obtained a pooled Receiver Characteristic Operator-Area Under the Curve (ROC-AUC) of $76.52 \%$ and $69.02 \%$ with the two strategies, respectively.

In Goel et al. (2020), the authors extracted discriminative features from the autoencoder and gray-level co-occurrence matrix using CT images. The obtained features are then combined with random forest classifier for COVID-19 detection. They achieved the following results: accuracy of $97.78 \%$, specificity of $98.77 \%$ and recall of $96.78 \%$.

Autoencoder can also be applied for survival chance detection of patients. As an example, Khozeimeh et al. (2021) combined CNN with autoencoder to predict survival chance of patients with positive COVID-19 diagnosis. In their experiments, clinical data are used such as blood pressure, liver disease, etc. The main problem facing this work is the data imbalance since the majority of infected patients are recovered (less mortality rate). To overcome this problem, data augmentation method is applied based on AE. Other autoencoder-based methods can be found in Berenguer et al. (2020), Shoeibi et al. (2020), Khobahi et al. (2020).

\subsection{Pretrained deep neural networks}

Pretrained models were originally trained on existing largescale labeled dataset (e.g., ImageNet) and later fine-tuned over the chest $\mathrm{CT}$ and X-ray images to accomplish the diagnosis process. The last layer in these models has been removed, and a new fully connected (FC) layer is added with an output size of two that represents two separate classes (COVID-19 or normal). In the obtained models, only the final FC layer is trained, while other layers are initialized with pretrained weights (Nayak et al. 2020). These models can be a very useful solution to the lack of large datasets for COVID-19. However, some challenges exist. One of the risen problems here is that the transfer across datasets from a domain to another can lead to deterioration of performance due to the gap existing between the domains. This is often the case with medical images taken from different centers. Moreover, there is an over-fitting problem with small amounts of COVID-19 datasets. Therefore, pretrained models are gener- 
ally used with some particular modifications in order to avoid the over-fitting problem.

In Nayak et al. (2020), eight pretrained CNN models have been compared including GoogLeNet, AlexNet, MobileNet-V2, VGG-16, SqueezeNet, ResNet-50; ResNet34 and Inception-V3. The obtained results for the classification of COVID-19 from normal cases show that ResNet-34 outperformed the other pretrained models and achieved an accuracy of $98.33 \%$. This evaluation has been conducted on a total of 286 scans of COVID-19 and normal classes as a training set, and 120 scans for the test $(60$ scans for each class). When dealing with the augmented dataset, the total of the training scans is 1002 , where 428 scans are used for the validation and 120 scans for the test. ResNet- 18 has been applied in Oh et al. (2020) using limited training datasets and achieved a sensitivity of $100 \%$ and $76.90 \%$ of precision for the COVID-19 class. Figures 9 and 10 show an example of AlexNet and VGG-16 architectures respectively. DenseNet201 pretrained model is used in Jaiswal et al. (2020) on chest CT images. To classify the patients into positive or negative COVID-19, deep transfer learning is carried out and obtained a training accuracy of $99.82 \%$ and validation accuracy of $97.48 \%$. The extreme version of the Inception (Xception) model is applied in Das et al. (2020) and achieved an accuracy of $97.40 \%$, f measure of $96.96 \%$, sensitivity of $97.09 \%$ and specificity of $97.29 \%$ for three classes COVID19 , pneumonia and other diseases.

Hemdan et al. (2020) proposed COVIDX-Net framework to diagnose the COVID-19 cases using X-ray images. It includes three main steps to accomplish the diagnostic process of the COVID-19 as follows: preprocessing, training model and validation, and classification. In consequence of the absence of public COVID-19 datasets, the experiments are carried out on 50 chest X-ray images where only 25 have been diagnosed with COVID-19, for the validation. The COVIDX-Net combined the following seven different architectures of deep CNN models: VGG-19, DenseNet121, InceptionV3, ResNetV2, Inception-ResNetV2, Xception and the second version of Google MobileNet. Figure 11 presents an overview of the COVIDX-Net framework. Another model called InstaCovNet-19 makes use of five pretrained models including Xception, ResNet101, InceptionV3, MobileNet and NASN is proposed in Gupta et al. (2020). Two classification strategies have been conducted : (COVID-19, pneumonia, normal) and (COVID, Non-COVID). Very high precision and classification accuracy have been achieved using the two strategies (see Table 2).

Similar method has been proposed by Narin et al. (2020) using five pretrained CNNs and three three different binary datasets including COVID-19, normal (healthy), bacterial and viral pneumonia patients. Gour and Jain (2020) proposed a new CNN model based on the VGG-19. They used a 30layered $\mathrm{CNN}$ model for the training with $\mathrm{X}$-ray images, and

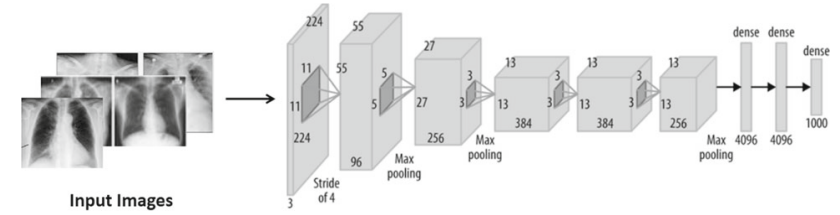

Fig. 9 AlexNet architecture proposed in Krizhevsky et al. (2017)

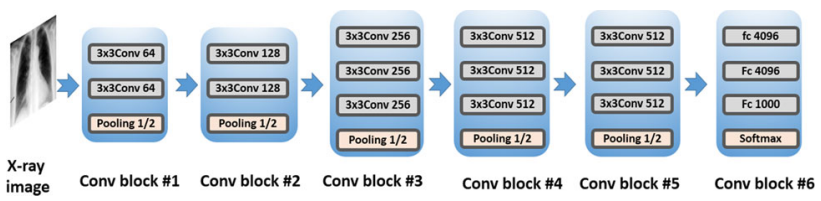

Fig. 10 VGG-16 architecture proposed in Simonyan and Zisserman (2014)

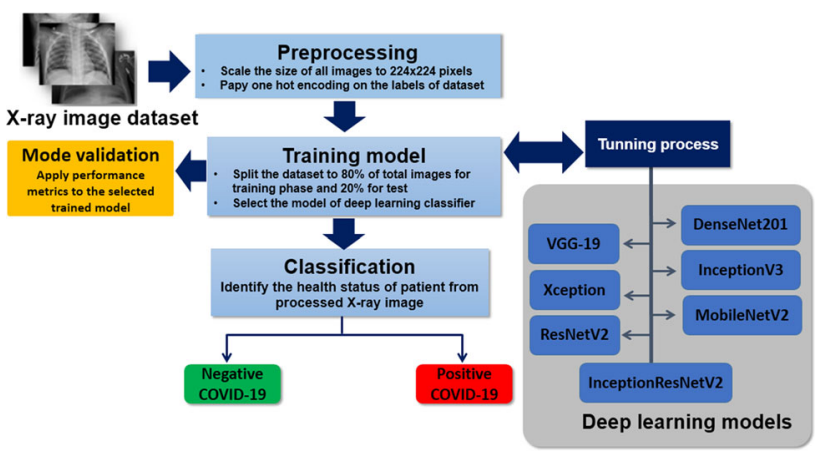

Fig. 11 COVIDX-Net framework Hemdan et al. (2020)

obtained sub-models using logistic regression. Other methods using pretrained CNNs can be found in Makris et al. (2020), Afshar et al. (2020), Kumar et al. (2020).

\subsection{D convolutional neural networks}

3D CNN models have also been used in the literature. They mainly extract 3D features from the segmented 3D lung region using CT images. For example, Wang et al. (2020e) segmented the lung region a pretrained UNet model. The obtained volumes were fed into the proposed DeCoVNet (3D deep convolutional neural Network to Detect COVID-19). A weakly supervised classification is then applied and achieved high COVID-19 classification performance and good lesion localization results. Müller et al. (2020) have also used UNet model instead of computational complex CNNs to reduce the over-fitting problem during the segmentation of the infected lung region. In Wang et al. (2020a), 3D-ResNet is applied for end-to-end training to classify the acquired lung images into pneumonia or healthy.

In order to predict the risk of COVID-19, Yang et al. (2020a) applied end-to-end training from CT images using the 3D Inception V1 model pretrained on the ImageNet dataset. The obtained accuracy was $95.78 \%$ overall and 
99.4\% on a part of the dataset containing 1,684 COVID-19 patients. Li et al. (2020) introduced a 3D DL system that aims to early detect the COVID-19, called COVNet. The COVNet model is composed essentially of ResNet50, which have a range of $\mathrm{CT}$ scans as entry and produces features for the equivalent scans. The obtained features from all scans are then involved by a max-pooling process. The final feature map is used as an input to a fully connected layer and softmax activation function to produce an output of a likelihood result for the three classes: COVID-19, non-pneumonia and community-acquired pneumonia (CAP). Han et al. (2020) introduced a deep 3D multiple instance learning to detect the COVID-19 using CT images. High accuracy has been achieved (97.9\%) and AUC of 99.0\%. Other 3D CNN-based methods can be found in de Vente et al. (2020), Liu et al. (2020).

\subsection{Combination of generic CNNs with traditional ML algorithms}

Another strategy is to use CNN models differently by combining them with traditional ML algorithms.

In Stephen et al. (2019), the authors presented a CNN model trained on X-ray images to recognize pneumonia. The proposed architecture consists of a combination of the convolution, max-pooling and rating layers. The obtained features comprise four convolutional layers, a max-pooling layer and a RELU activator between them. The traditional ML algorithm ANN (artificial neural network) is finally applied for classification. ANN and AlexNet architecture have been combined in Aslan et al. (2020) to systematically find out COVID-19 pneumonia subjects using CT scans. Firstly, a segmentation using ANN algorithm is performed to localize the lungs. Next, COVID-19 classes are augmented to produce more images. Finally, pretrained AlexNet architecture is used in one time with only a transfer learning process, the obtained accuracy is $98.14 \%$. And with additional layer called Bidirectional Long Short-Term Memories in the second time, with an accuracy $98.70 \%$, Nour et al. (2020) proposed a scratch CNN model including five convolution layered serial network. Three ML algorithms have been trained on the obtained deep features involving k-NN, SVM and DT. The highest accuracy is obtained by the SVM with $98.97 \%$.

Instead of using pretrained deep CNNs only as feature extractor, in Ismael and Şengür (2020), two other strategies have been conducted to accurately classify Chest X-ray images into positive of negative COVID-19 including finetuning strategy and end to end training. The following models have been used as a feature extractor : ResNet101, VGG19, ResNet50, ResNet18 and VGG16 where SVM is used for ML-based classification, whereas a new CNN model is used for the fine-tuning strategy. Finally, end-to-end training with a dataset of 180 COVID-19 and 200 normal is car- ried out as a third strategy. $94.7 \%$ of accuracy is achieved using ResNet50 model and SVM classifier, where fine-tuned strategy with ResNet50 model achieved $92.6 \%$. Finally, the end-to-end training strategy of the developed CNN model realized a $91.6 \%$ result. Deep CNN and long short-term memory (LSTM) have been combined in Islam et al. (2020) to diagnose COVID-19 automatically from X-ray images. The obtained accuracy of the classification of three classes (COVID-19, normal and other pneumonia) is $99.4 \%$. Similar methods that combine deep features and classical ML techniques can be found in Sethy and Behera (2020).

Sharifrazi et al. (2021) proposed to improve the 2D-CNN's performance by applying a Sobel filter to detect COVID19 from a new collected dataset of X-ray images. An SVM algorithm is then employed to classify the input images. This proposed method has shown a high performance with not many data.

\subsection{Ensemble models}

Handling the problem of COVID-19 detection using a single DL model without any specific addition might not achieve a high accuracy classification using CXR images or CT scans. For this reason, the use of many DL models combined with each other can be a good solution, namely ensemble model, and the learning approach is called ensemble learning. For example, the authors in Sitaula and Hossain (2020) proposed a DL model, namely attention-based VGG-16. This model used VGG-16 to capture the spatial relationship between the ROIs in CXR images. By using an appropriate convolution layer (4th pooling layer) of the VGG-16 model in addition to the attention module, they added a novel DL model to perform fine-tuning in the classification process. In Hall et al. (2020) ensemble of three pretrained models including ResNet50 and VGG16 and an own small CNN is applied for a test set of 33 new COVID-19 and 218 pneumonia cases. The overall accuracy realized is of $91.24 \%$. Shalbaf et al. (2020) an ensemble deep transfer learning system with 15 pretrained $\mathrm{CNN}$ architectures on CT images. They obtained the following results: accuracy (85\%), precision $(85.7 \%)$ and recall $(85.2 \%)$.

\subsection{Smartphone applications}

To further automate the screening of COVID-19 and to make it faster, mobile phones can be a very interesting framework for that due to their facility and numerous sensors with important computing proficiencies. Specifically, a smartphone has is able to scan CT images of COVID-19 patients to use them for analysis screening. Moreover, multiple CT images of the same COVID-19 patient can be gathered into one smartphone for similarity examination of how disfigurement have been developed (Purswani et al. 2019). However, the com- 
puting capability of a mobile to treat a large amount of data is lower than a grand machine or a computer. Therefore, a lightweight representation is needed to accomplish this task. Consequently, various recent methods have been proposed to detect COVID-19 in mobile devices using a slight representation. In Zulkifley et al. (2020), a lightweight DL model namely LightCovidNet has been offered to detect COVID-19 using a mobile platform. To enhance the performance of the proposed model, supplementary data have been generated and added to the training dataset using the conditional deep convolutional GAN. In order to reduce the memory usage of the proposed model, five units of feed-forward CNN are built using separable convolution operators. Multi-scale features are then learned to be suitable for the X-ray images which have been acquired from all over the world separately. Instead of COVID-19 diagnosis and detection, various lightweight applications have been introduced to delay the spread of the virus. These applications could be designed to be compatible with the capabilities of a smartphone to further speed up their operation. Among these applications, we can find masked face recognition (Hariri 2020), facial mask detection (Chen et al. 2020; Chua et al. 2020), social distance monitoring (Ahmed et al. 2020; Rezaei and Azarmi 2020) and human mobility estimation (Xiong et al. 2020). Other mobile-based technique using to fight against COVID-19 has been proposed in Maghdid et al. (2020). Using DL algorithms, the authors arrived to efficiently evaluate the level of pneumonia and thus to determine whether it is a COVID-19 case or not.

\section{Deep learning for other applications}

Other applications have been proposed to fight against COVID-19-induced pneumonia; the most important are cough detection and human mobility estimation.

\subsection{Cough detection}

In addition to the DL approaches using X-ray and chest CT scans for COVID-19 detection, scientists affirm that audio sounds generated by the respiratory system can be diagnosed and analyzed to decide whether the patient is infected or not. Therefore, cough analysis has been used to screen and diagnose COVID-19. ML techniques can supply useful cues enabling the development of a diagnostic instrument. To do so, cough data of COVID and non-COVID are required. Accordingly, Sharma et al. (2020) proposed a database called Coswara, of respiratory sounds, namely cough, breath and voice. Some experiments have been recently carried out to screen COVID-19 from acoustic features; for example, in Hassan et al. (2020), recurrent neural network (RNN) has been used in its new architecture, namely the Long ShortTerm Memory (LSTM) to extract six speech features from

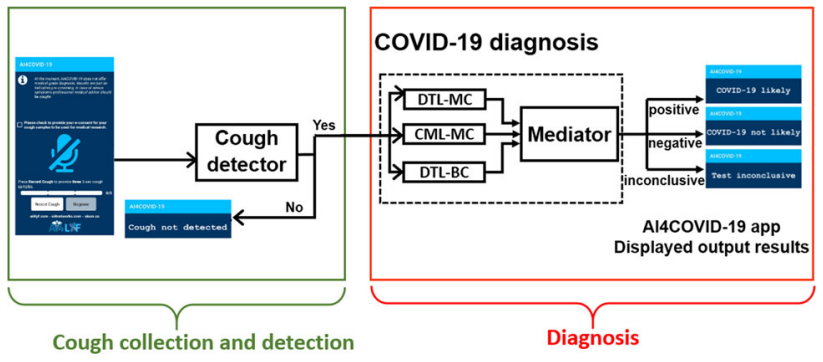

Fig. 12 AI4COVID-19 system architecture Imran et al. (2020)

a collected dataset (i.e., spectral centroid, spectral roll-off, zero-crossing rate, zero-crossing rate, MFCC and MFCC). In this work, $70 \%$ of the data were used for training and 30\% for testing. The obtained results show that the best accuracy is achieved for breathing sound, reaching up to $98.2 \%$ followed by cough sounds; an accuracy of $97 \%$ is attained, whereas the accuracy of voice analysis is of $88.2 \%$.

A smartphone application using cough-based diagnosis for COVID-19 detection is proposed in Imran et al. (2020). This application is based on an AI-powered screening solution called AI4COVID-19. Its principle is to send three 3 second cough sounds to an AI engine running in the cloud and give a result during two minutes. To overcome the lack of COVID-19 cough training data, the authors applied transfer learning using ESC-50 dataset (Piczak 2015) that contains 50 classes of cough and non-cough sounds acquired using a smartphone. Figure 12 presents the offered system architecture and a drawing of the AI4COVID-19. The obtained results show high overall accuracy of $95.60 \%$, a sensitivity of $96.01 \%$, a specificity of $95.19 \%$ and precision of $95.22 \%$. Schuller et al. (2020) studied what computer audition could possibly contribute to the ongoing battle against the COVID-19. Other recent acoustic analyses for the detection of COVID-19 can be found in Deshpande and Schuller (2020), Alsabek et al. (2020), Pal and Sankarasubbu (2020), Quatieri et al. (2020), Laguarta et al. (2020), Deshmukh et al. (2020).

\subsection{Estimating human mobility}

Human mobility (movement) is one of the main factors that promote the transmission of the virus. Policy-makers find huge difficulties to find an optimal protocol to insure the social distancing and barrier measures. To solve this problem, Bao et al. (2020) proposed a system that aims to evaluate and estimate maps of people movement responses by learning from existing ground truth data. The proposed system is based on a DL based-data generation called COVIDGAN. It merges a diversity of features involving contextual features, COVID-19 details and data history, as well as policies from various origins such as news, reports and Safe- 
Graph. Experiment results showed that COVID-GAN can well imitate real-world human movement reactions and the area-constraint-based correction can considerably upgrade the solution value. To further explain the relation between people mobility and COVID-19 contamination, Xiong et al. (2020) presented a study using mobile device data to give more insights to decision makers about the national mobility tendencies before and during the pandemic.

\subsection{Forecasting of new cases and new deaths}

Along with the detection approaches presented above, forecasting of new cases and new deaths has been studied recently in order to help the governments take right decisions during this pandemic. Therefore, COVID-19 time series predicting have been studied using DL techniques, one of the most used models is LSTM that has been introduced by Hochreiter and Schmidhuber (1997) and applied by Wang et al. for COVID-19 time series prediction (Wang et al. 2020c). LSTM is designed to combine the short-term and long-term temporal data and provides accurate time series forecasting. For example, Ayoobi et al. (2021) have used LSTM, convolutional LSTM and GRU to predict new cases and new deaths during the COVID-19 crisis. Bi-directional models have been employed for the prediction; it is considered as an improved LSTM. It consists of using the traditional LSTM model to compute the input information at subsequent and reverse order to obtain 2 different exterior outputs and get the final output using a fully associated layer.

\section{Available COVID-19 datasets description}

Since the spread of the virus from Wuhan to many countries in the world, many COVID-19 datasets have been introduced in the literature in order to apply the deep learning approaches for automatic detection of the virus. In the following, we give the newly available datasets where the images are X-ray or CT scans.

\subsection{X-ray datasets}

Many X-ray datasets have been presented to boost the DL techniques for COVID-19 detection. Here, we present two examples.

COVID-19 Radiography Database the initial dataset is divided into three different folders (i.e., training, testing and validation) and three sub-folders containing COVID-19, viral pneumonia and normal chest $\mathrm{X}$-ray images, respectively (Rahman 2020). We have used the version 4 of this database that contains 3616 COVID-19-positive cases along with 10,192 normal, 6012 lung opacity (non-COVID lung infection) and 1345 viral pneumonia images. In this work,

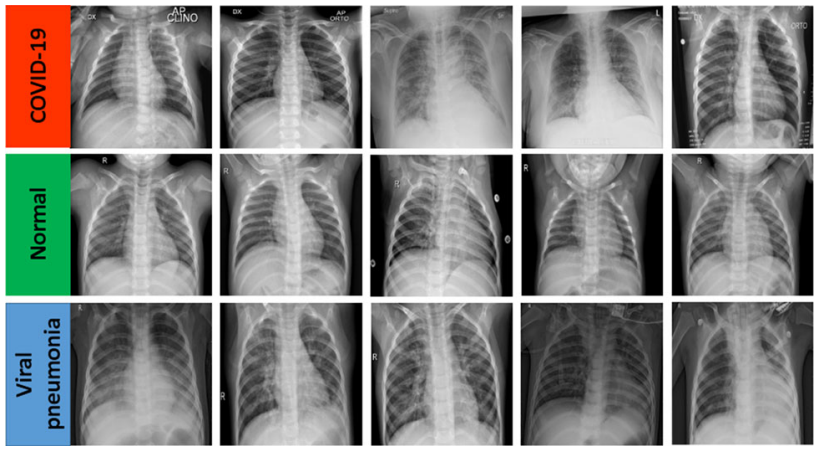

Fig. 13 X-ray images from COVID-19 Radiography Database Rahman (2020)

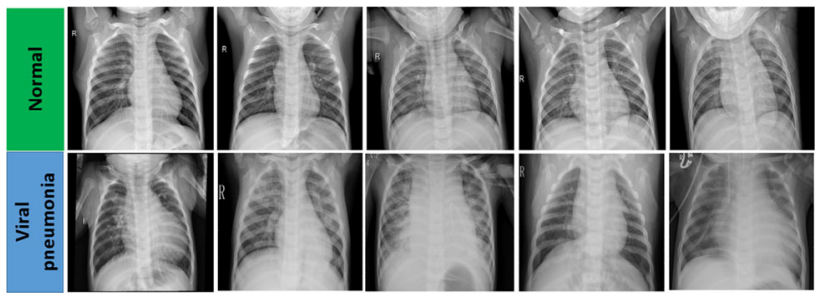

Fig. 14 X-ray images diagnosed COVID-19 from Covid chestxray dataset master Cohen et al. (2020)

we carry out the classification of only three classes (COVID19, normal, VP). Some scans from this database are shown in Fig. 13.

Covid chestxray dataset master Cohen et al. (2020) is a new and challenging dataset presented to assess the COVID19 detection systems. It is one of the first introduced datasets during the COVID-19 pandemic. This dataset contains 5856 $\mathrm{X}$-ray images divided into three parts (train, test and validation). The train part contains 1341 normal images and 3875 pneumonia, whereas the test part contains 234 of normal cases and 390 pneumonia. We can notice that this dataset unbalanced, this makes it more challenging since more adjustments are required to avoid the biased class problem. The chest X-ray images are acquired from hospitals with a frontal view. Considering the Covid chestxray dataset master as a benchmark is very tricky because of its sort of composition. Most of the proposed methods using this dataset for two-class classification problem. Some images of COVID-19 cases from this database are shown in Fig. 14.

\subsection{CT scan datasets}

CT scan datasets have been mostly introduced to segment specific regions in the thorax in order to diagnose COVID19 patients. Here, we present one of these datasets.

The COVID-CT-Dataset contains 349 CT scan images diagnosed COVID-19 from 216 patients (Yang et al. 2020b; Zhao et al. 2020). The authors have based on many reposi- 


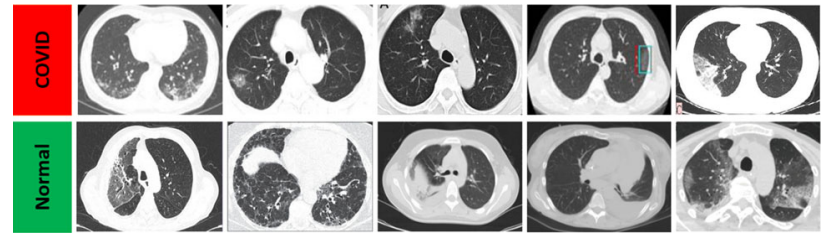

Fig. 15 CT scans from COVID-CT-Dataset

tories (i.e., medRxiv2 and bioRxiv3) to extract these images from 760 preprints. To associate each image to the right class (e.g., COVID, PV, normal), the captions of the corresponding figures are used along with PyMuPDF4 to get low-level structure data. The quality of the images extracted from the PDF files has been maintained. In the case of not clear caption, the meta-data of the paper has been used to associate the images to its label. Some images of COVID-CT-Dataset are presented in Fig. 15.

CT Scan COVID Prediction contains 746 CT scan images divided into train set (500 images) and test set (246 images) (He et al. 2020). These images have been collected from many papers in the context of COVID19 prediction and segmentation from ArXiv, medRxiv, NEJM, etc. This database is suitable for deep learning methods and enables easy diagnose of COVID-19 using CT scans.

Other X-ray and CT scan databases can be found in Table 1.

\section{Discussion}

Table 2 includes some studies conducted with DL models. It can be seen in Table 1 that studies have focused on two popular images, namely CT and X-ray images. The most used of these is X-ray images. This is because it is easily available everywhere. In addition, both its low memory space and high results in its performance encouraged researchers to use $\mathrm{X}$-ray images. In addition, the greater availability of X-ray data from COVID-19 patients in public databases has led to the large number of these studies. In articles in the field of medicine, it is often stated that CT images show higher performance. However, these high accuracies are not seen in DL based CAD systems. This may be because radiologists can easily distinguish patients from CT images. CT images have too many cross-sections of the same person. They are much more complex than X-ray images. This complex situation is a disadvantage in distinguishing them in DL methods. The combination of X-ray and CT images, performed in few studies, also shows that it gives good results. It can be said that studies are carried out with multi-class solutions rather than the binary classification problem. It is seen that studies carried out with 3D data have lower performance. It has mostly been studied with 2D data. The results obtained from

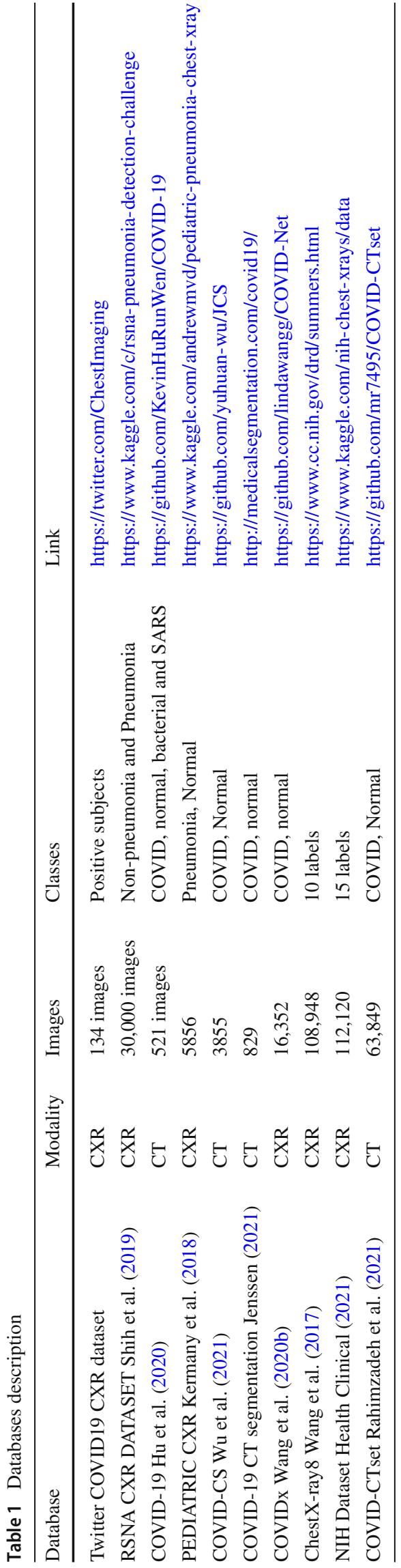




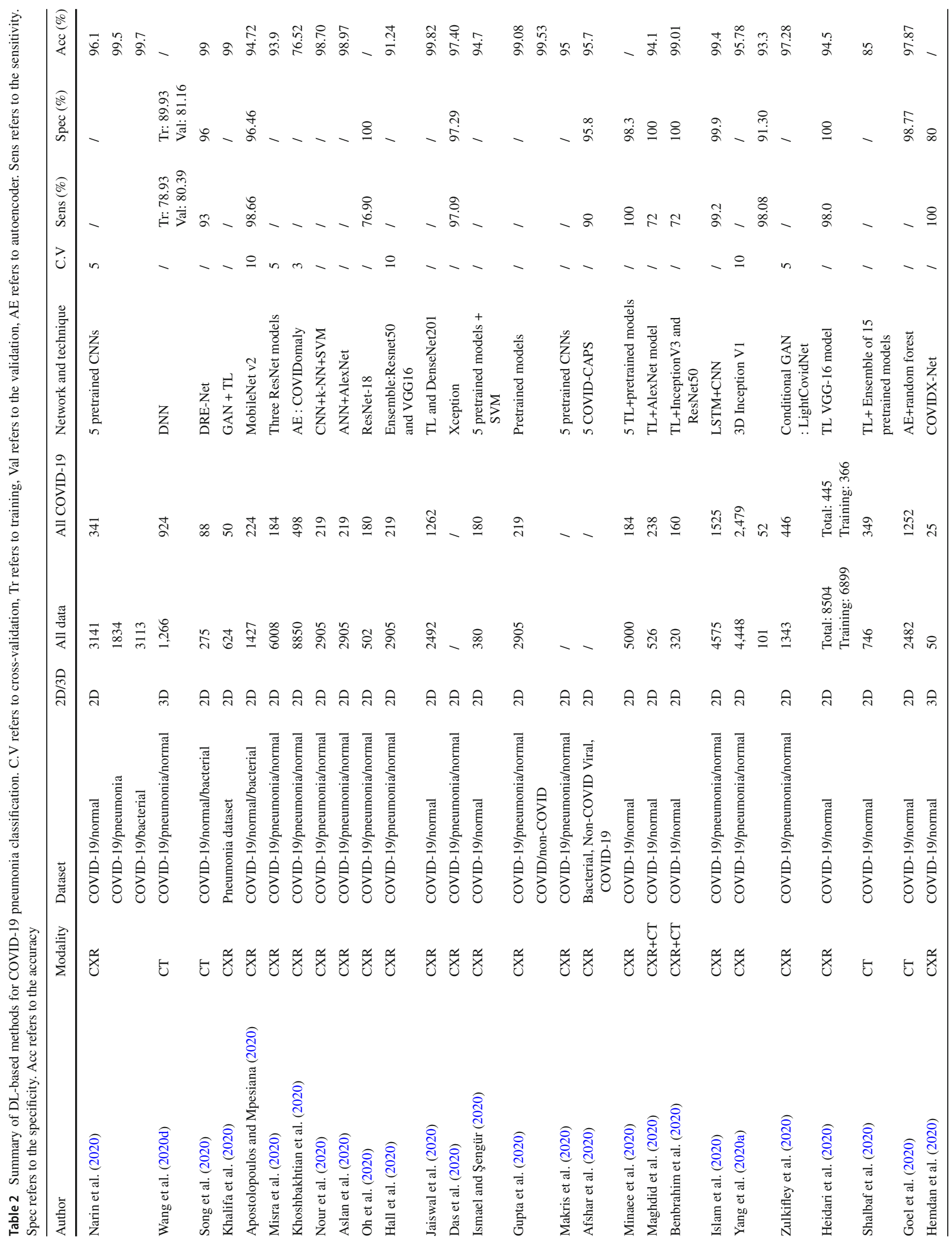


these data are very high. Considering the number of data, it is known that DL models work stably with a lot of data. According to this fact, the number of data used is not sufficient. It can be stated that this is one of the biggest problems in the detection of COVID-19. It is very important to compare the studies conducted with the data obtained from many different centers. Otherwise, the accuracy of the studies can be fooled. For this, we recommend that data collected from many different centers be offered to researchers from a single center. However, in studies conducted with a small number of data, it is seen that pretrained models are used to ensure high model training. Especially, Keras, TensorFlow, PyTorch and MATLAB also include these pretrained models. The biggest problem here is that these models are trained with the ImageNet dataset. Having a lot of data belonging to very different classes in the ImageNet dataset can reduce the trust in these models. However, it is seen that the performances obtained are also very high.

Traditional ML algorithms are also used by using the feature extraction part of DL models. This approach appears to improve performance. It is generally seen that the SVM algorithm is used. Most of the studies do not use any crossvalidation $(\mathrm{CV})$ method. We think that this decreases the reliability of the results. Because it is not known how the test data are distinguished. A very high performance test dataset can be created. This overestimates the results obtained. It can be noted that a CV method should be used in which the whole data set can be tested in studies. Although there are many studies using DL-based methods, it is very difficult to produce sufficiently transparent, stable and reliable models. It was clearly stated above that there are many parameters affecting the results.

As an alternative to the studies performed on X-ray and CT images, researches on the detection of COVID-19 are carried out with sound and cough-based acoustic sound analysis. Alternative approaches are very important in the detection of COVID-19. It may be possible to save more lives with realtime detection and diagnosis systems with online scanning systems installed with mobile or computer. As a result of all these approaches, namely RT-PCR test, DL-based X-ray and CT images, detection and acoustic examinations, it is predicted that patients with COVID-19 can be detected more stable and with higher accuracy. Because, considering that the epidemic started with a person, it is very important to correctly diagnose even a person.

\section{Future challenges on the detection of COVID-19}

Despite the good performances achieved by the deep learning techniques along with X-ray images and CT scans to detect the virus, there are still many challenges to be addressed.
Figure 16 presents the number of modalities used by the reviewed papers. It is clear that X-ray images are the most used and the more available. Nevertheless, it is clear that very few researchers have used combined modalities of X-ray and CT images due to the absence of such a comprehensive database. Moreover, these images don't provide any additional information about the patients like gender and age. Incorporating this information along with manual or ground truths, however, could improve the efficiency of DL-based methods.

Forecasting research is also very limited and will be more challenging especially because the virus mutations are not known. Hence, predicting its spread, mortality and symptoms as well as its relation with the weather are still very tricky tasks.

Moreover, since COVID-19 detection is becoming a hot research area by multidisciplinary research, a large amount of heterogeneous data including results and analysis are increasingly available. These data are generally acquired from different sources (e.g., clinical, behavior, physiology and pharmacology data). In order to make this data useful, it should be analyzed to extract knowledge. This is one of the most challenging tasks that could be solved using data mining and machine learning techniques. Cinaglia et al. (2019) present some solutions to the aforementioned challenge in life science to extract knowledge using machine learning and data mining algorithms for data analysis.

Also, when policy-makers and citizens are making their best to submit to the difficult constraints of lockdown and social distancing, AI can be used to create more intelligent robots and autonomous machines to help health workforce and to reduce their workload by disinfection, working in hospitals, food distributing and helping the patients. The challenge of this solution is that people lack confidence in autonomous machines and prefer to be served by a human even if there is a risk of virus transmission. Moreover, entrusting chatbots to diagnose patients needs a large amount of medical data from experts.

Besides, the difference in languages from a country to another makes an already difficult task still more arduous. On the other hand, when dealing with voice analysis, there are still many challenges to be taken up. For example, until now, annotated data of patients' voices are not publicly available for research purposes of COVID-19 detection and diagnosis. Collecting these data is mostly made in unconstrained environments (i.e., in-the-wild) using smartphones or other voice recorders. These environments are generally noisy and contain reverberation, which leads to bad quality of data and makes the diagnosis and detection of COVID-19 more challenging. Also, one of the most important future challenges is to concentrate on further decreasing the false negative rate and, as far as practicable, reducing the false positive rate by the same token to accurately differentiate viral from BP. 


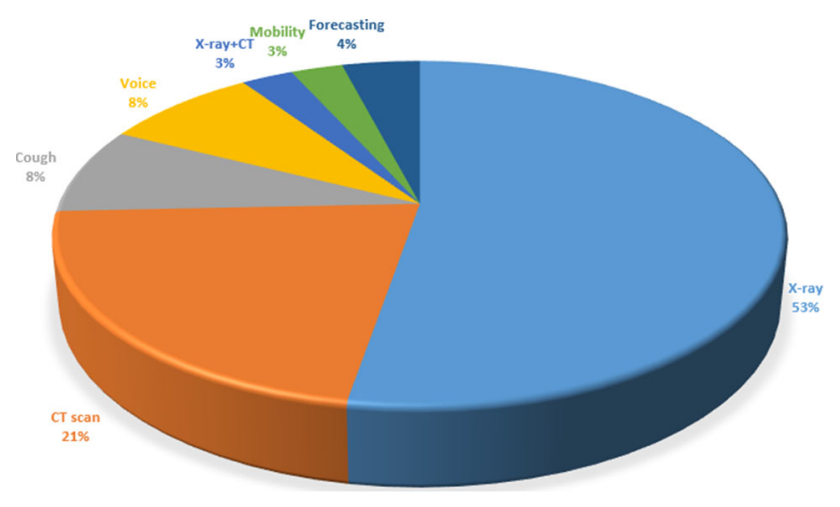

Fig. 16 Number of modalities used to detect or predict COVID-19 using deep learning methods. The rates are computed from the reviewed papers

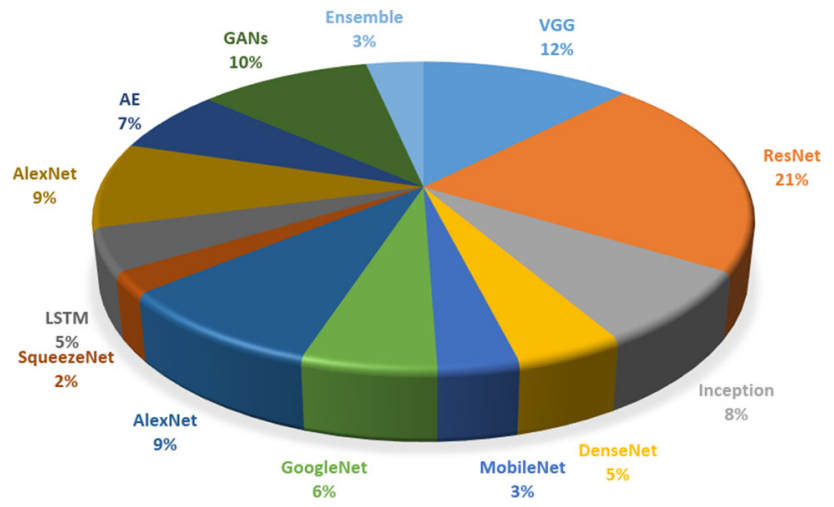

Fig. 17 Number of pretrained models used to detect or predict COVID19. The rates are computed from the reviewed papers

Although environments like Google Colab and Amazon have relatively solved the lack of powerful hardware to implement deep learning architectures with GPUs Graphic Cards, enrolling these environments in real medical laboratories is still a very challenging task.

\section{Conclusion}

Although the RT-PCR test is considered the gold standard for COVID-19 diagnosis, it is time-consuming to make a decision because of high false-negative levels in the results. Therefore, medical imaging modalities such as chest X-ray and chest $\mathrm{CT}$ scans are the best alternative according to scientists. Chest X-ray radiography is of low cost and low radiation dose, it is available and easy to use in general or community hospitals. This review presents a detailed study of the existing solutions that are mainly based on DL techniques to early diagnose the COVID-19. This study gives more of an insight into the scientists' and decision-makers' thought processesnot only during the wave periods but also during that of the vaccination that could require real-time mass testing. The lack of data, however, is the mandatory problem to achieve efficient and real-time results. Many solutions have been presented and discussed in this review study to give more ideas to future trends and also for eventual future diseases that might suffer from the missing-data problem. We believe that with more public databases, better DL based-approaches can be developed to detect and diagnose the COVID-19 accurately.

According to the statistics provided in Shoeibi et al. (2020), the majority of research are devoted to the detection of COVID-19 where due to a lack of publicly accessible databases, limited research are done on forecasting. Also, from this review paper, we can notice that many pretrained CNNs have been employed to deal with COVID-19 detection or prediction as shown in Fig. 17; it is difficult to make a fair comparison between their performance since their methods apply different protocols and combine many different datasets to enrich the DL model. Accordingly, although transfer learning these pretrained models has shown very high performance, it is not easy to decide which one is more suitable for COVID-19 detection or forecasting.

Acknowledgements The authors would like to thank the 'Agence Nationale de Valorisation des Résultats de la Recherche et du Développement Technologique (DGRSDT), Algérie'.

\section{Declarations}

Conflict of interest The authors declare that they have no conflict of interest.

\section{References}

Abbas A, Abdelsamea MM, Gaber MM (2020) Classification of covid19 in chest $\mathrm{x}$-ray images using detrac deep convolutional neural network. arXiv:2003.13815

Abenavoli L, Cinaglia P, Luzza F, Gentile I, Boccuto L (2020) Epidemiology of coronavirus disease outbreak: the Italian trends. Rev Recent Clin Trials 15(2):87-92

Afshar P, Heidarian S, Naderkhani F, Oikonomou A, Plataniotis KN, Mohammadi A (2020) Covid-caps: a capsule network-based framework for identification of covid-19 cases from x-ray images. arXiv:2004.02696

Ahmed I, Ahmad M, Rodrigues JJ, Jeon G, Din S (2020) A deep learning-based social distance monitoring framework for covid19. Sustainable Cities and Society p. 102571

Ahmed S, Hossain MF, Noor MBT (2021) Convid-net: an enhanced convolutional neural network framework for covid-19 detection from x-ray images. In: Proceedings of international conference on trends in computational and cognitive engineering, pp 671-681. Springer

Ai T, Yang Z, Hou H, Zhan C, Chen C, Lv W, Tao Q, Sun Z, Xia L (2020) Correlation of chest ct and rt-pcr testing in coronavirus disease 2019 (covid-19) in China: a report of 1014 cases. Radiology p 200642

Al-Tawfiq JA (2020) Asymptomatic coronavirus infection: mers-cov and sars-cov-2 (covid-19). Travel Med Infectious Dis 35:101608 
Alizadehsani R, Sharifrazi D, Izadi NH, Joloudari JH, Shoeibi A, Gorriz JM, Hussain S, Arco JE, Sani ZA, Khozeimeh F, et al (2021) Uncertainty-aware semi-supervised method using large unlabelled and limited labeled covid-19 data. arXiv:2102.06388

Alsabek MB, Shahin I, Hassan A (2020) Studying the similarity of covid-19 sounds based on correlation analysis of mfcc. In: 2020 international conference on communications, computing, cybersecurity, and informatics (CCCI), pp 1-5. IEEE

Apostolopoulos ID, Mpesiana TA (2020) Covid-19: automatic detection from $x$-ray images utilizing transfer learning with convolutional neural networks. Phys Eng Sci Med 43:635-640

Aslan MF, Unlersen MF, Sabanci K, Durdu A (2020) Cnn-based transfer learning-bilstm network: a novel approach for covid-19 infection detection. Appl Soft Comput 98:106912

Ayoobi N, Sharifrazi D, Alizadehsani R, Shoeibi A, Gorriz JM, Moosaei H, Khosravi A, Nahavandi S, Chofreh AG, Goni FA, et al. (2021) Time series forecasting of new cases and new deaths rate for covid19 using deep learning methods. arXiv:2104.15007

Baldi P (2012) Autoencoders, unsupervised learning, and deep architectures. In: Proceedings of ICML workshop on unsupervised and transfer learning, pp 37-49

Bao H, Zhou X, Zhang Y, Li Y, Xie Y (2020) Covid-gan: estimating human mobility responses to covid-19 pandemic through spatio-temporal conditional generative adversarial networks. In: Proceedings of the 28th international conference on advances in geographic information systems, pp 273-282

Benbrahim H, Hachimi H, Amine A (2020) Deep transfer learning with apache spark to detect covid-19 in chest X-ray images. Rom J Inf Sci Technol 23:S117-S129

Berenguer AD, Sahli H, Joukovsky B, Kvasnytsia M, Dirks I, AlioschaPerez M, Deligiannis N, Gonidakis P, Sánchez SA, Brahimetaj $\mathrm{R}$, et al. (2020) Explainable-by-design semi-supervised representation learning for covid-19 diagnosis from ct imaging. arXiv:2011.11719

Chen Y, Hu M, Hua C, Zhai G, Zhang J, Li Q, Yang SX (2020) Face mask assistant: detection of face mask service stage based on mobile phone. arXiv:2010.06421

Chouhan V, Singh SK, Khamparia A, Gupta D, Tiwari P, Moreira C, Damaševičius R, De Albuquerque VHC (2020) A novel transfer learning based approach for pneumonia detection in chest x-ray images. Appl Sci 10(2):559

Chua MH, Cheng W, Goh SS, Kong J, Li B, Lim JY, Mao L, Wang S, Xue K, Yang L et al (2020) Face masks in the new COVID-19 normal: materials, testing, and perspectives. Research 2020:7286735. https://doi.org/10.34133/2020/7286735

Cinaglia P, Mirarchi D, Veltri P (2019) Information retrieval in life sciences. Ref Mod Life Sci 1104-1108

Cinaglia P, Tradigo G, Cascini GL, Zumpano E, Veltri P (2018) A framework for the decomposition and features extraction from lung dicom images. In: Proceedings of the 22nd international database engineering \& applications symposium, pp 31-36

Cohen JP, Morrison P, Dao L, Roth K, Duong TQ, Ghassemi M (2020) Covid-19 image data collection: prospective predictions are the future. arXiv:2006.11988

Das NN, Kumar N, Kaur M, Kumar V, Singh D (2020) Automated deep transfer learning-based approach for detection of Covid-19 infection in chest X-rays. IRBM. https://doi.org/10.1016/j.irbm. 2020.07.001

Deshmukh S, Ismail MA, Singh R (2020) Interpreting glottal flow dynamics for detecting covid-19 from voice. arXiv:2010.16318

Deshpande G, Schuller BW (2020) Audio, speech, language, \& signal processing for covid-19: a comprehensive overview. arXiv:2011.14445

de Vente C, Boulogne LH, Venkadesh KV, Sital C, Lessmann N, Jacobs C, Sánchez CI, van Ginneken B (2020) Improving automated covid-19 grading with convolutional neural networks in computed tomography scans: an ablation study. arXiv:2009.09725

Fu M, Yi SL, Zeng Y, Ye F, Li Y, Dong X, Ren YD, Luo L, Pan JS, Zhang Q (2020) Deep learning-based recognizing covid-19 and other common infectious diseases of the lung by chest ct scan images. medRxiv

Ghassemi N, Shoeibi A, Khodatars M, Heras J, Rahimi A, Zare A, Pachori RB, Gorriz JM (2021) Automatic diagnosis of covid-19 from ct images using cyclegan and transfer learning. arXiv:2104.11949

Ghosh A, Saha S (2018) Automatic identification of fracture region within bone in x-ray image. In: 2018 2nd international conference on electronics, materials engineering \& nano-technology (IEMENTech), pp 1-7. IEEE

Goel C, Kumar A, Dubey SK, Srivastava V (2020) Efficient deep network architecture for covid-19 detection using computed tomography images. medRxiv

Gour M, Jain S (2020) Stacked convolutional neural network for diagnosis of covid-19 disease from X-ray images. arXiv:2006.13817

Gozes O, Frid-Adar M, Greenspan H, Browning PD, Zhang H, Ji W, Bernheim A, Siegel E (2020) Rapid ai development cycle for the coronavirus (covid-19) pandemic: initial results for automated detection \& patient monitoring using deep learning ct image analysis. arXiv:2003.05037

Group CIS (2021) mHistoric 21-day lockdown, predictions for lockdown effects and the role of data in this crisis of virus in India. "https://bhramarm.medium.com/historic-lockdown-predictionmodels-to-study-lockdown-effects-and-the-role-of-data-in-thecrisis-a0afeeec5a6". Accessed 01 May 2021

Grubaugh ND, Hanage WP, Rasmussen AL (2020) Making sense of mutation: what $\mathrm{d} 614 \mathrm{~g}$ means for the covid-19 pandemic remains unclear. Cell 182(4):794-795

Gupta A, Gupta S, Katarya R et al (2020) Instacovnet-19: a deep learning classification model for the detection of covid-19 patients using chest x-ray. Appl Soft Comput 99:106859

Haghanifar A, Majdabadi MM, Ko S (2020) Covid-cxnet: detecting covid-19 in frontal chest $\mathrm{x}$-ray images using deep learning. arXiv:2006.13807

Hall LO, Paul R, Goldgof DB, Goldgof GM (2020) Finding covid19 from chest $\mathrm{x}$-rays using deep learning on a small dataset. arXiv:2004.02060

Han Z, Wei B, Hong Y, Li T, Cong J, Zhu X, Wei H, Zhang W (2020) Accurate screening of covid-19 using attention based deep 3d multiple instance learning. IEEE Trans Med Imag 39:2584-2594

Hariri W (2020) Efficient masked face recognition method during the covid-19 pandemic. arXiv:2105.03026

Hassan A, Shahin I, Alsabek MB (2020) Covid-19 detection system using recurrent neural networks. In: 2020 International conference on communications, computing, cybersecurity, and informatics (CCCI), pp 1-5. IEEE

He X, Yang X, Zhang S, Zhao J, Zhang Y, Xing E, Xie P (2020) Sampleefficient deep learning for covid-19 diagnosis based on ct scans. medrxiv

of Health Clinical Center, NI (2021) Nih chest x-ray dataset of 14 common thorax disease categories. https://www.kaggle.com/nihchest-xrays/data. Accessed 25 July 2021

Heidari M, Mirniaharikandehei S, Khuzani AZ, Danala G, Qiu Y, Zheng B (2020) Improving the performance of cnn to predict the likelihood of covid-19 using chest $\mathrm{x}$-ray images with preprocessing algorithms. Int J Med Informatics 144:104284

Hemdan EED, Shouman MA, Karar ME (2020) Covidx-net: a framework of deep learning classifiers to diagnose covid-19 in x-ray images. arXiv:2003.11055

Heneghan C (2020) Differentiating viral from bacterial pneumonia (2020). https://www.cebm.net/covid-19/differentiating-viralfrom-bacterial-pneumonia. Accessed 07 Dec 2020 
Hochreiter S, Schmidhuber J (1997) Long short-term memory. Neural Comput 9(8):1735-1780

Hu R, Ruan G, Xiang S, Huang M, Liang Q, Li J (2020) Automated diagnosis of covid-19 using deep learning and data augmentation on chest ct. medRxiv

Huang P, Liu T, Huang L, Liu H, Lei M, Xu W, Hu X, Chen J, Liu B (2020) Use of chest ct in combination with negative rt-pcr assay for the 2019 novel coronavirus but high clinical suspicion. Radiology 295(1):22-23

Imran A, Posokhova I, Qureshi HN, Masood U, Riaz S, Ali K, John CN, Nabeel M (2020) Ai4covid-19: Ai enabled preliminary diagnosis for covid-19 from cough samples via an app. arXiv:2004.01275

Islam MZ, Islam MM, Asraf A (2020) A combined deep cnn-lstm network for the detection of novel coronavirus (covid-19) using X-ray images. Informatics Med Unlock 20:100412

Ismael AM, Şengür A (2020) Deep learning approaches for covid19 detection based on chest x-ray images. Expert Syst Appl 164:114054

Jaiswal A, Gianchandani N, Singh D, Kumar V, Kaur M (2020) Classification of the covid-19 infected patients using densenet201 based deep transfer learning. J Biomol Struct Dyn, pp 1-8

Jaiswal AK, Tiwari P, Rathi VK, Qian J, Pandey HM, Albuquerque VHC (2020) Covidpen: a novel covid-19 detection model using chest X-rays and ct scans. medRxiv. https://doi.org/10.1101/2020. 07.08.20149161

Javan AAK, Jafari M, Shoeibi A, Zare A, Khodatars M, Ghassemi N, Alizadehsani R, Gorriz JM (2021) Medical images encryption based on adaptive-robust multi-mode synchronization of chen hyper-chaotic systems. Sensors 21(11):3925

Jenssen HB (2021) COVID-19 CT segmentation dataset. http:// medicalsegmentation.com/covid19/. Accessed 25 July 2021

Karakanis S, Leontidis G (2020) Lightweight deep learning models for detecting covid-19 from chest x-ray images. Tech. rep, EasyChair

Kermany DS, Goldbaum M, Cai W, Valentim CC, Liang H, Baxter SL, McKeown A, Yang G, Wu X, Yan Fet al (2018) Identifying medical diagnoses and treatable diseases by image-based deep learning. Cell 172(5):1122-1131

Khalifa NEM, Taha MHN, Hassanien AE, Elghamrawy S (2020) Detection of coronavirus (covid-19) associated pneumonia based on generative adversarial networks and a fine-tuned deep transfer learning model using chest x-ray dataset. arXiv:2004.01184

Khobahi S, Agarwal C, Soltanalian M (2020) Coronet: a deep network architecture for semi-supervised task-based identification of covid19 from chest $\mathrm{x}$-ray images. medRxiv

Khoshbakhtian F, Ashraf AB, Khan SS (2020) Covidomaly: a deep convolutional autoencoder approach for detecting early cases of covid-19. arXiv:2010.02814

Khozeimeh F, Sharifrazi D, Izadi NH, Joloudari JH, Shoeibi A, Alizadehsani R, Gorriz JM, Hussain S, Sani ZA, Moosaei H, et al. (2021) Cnn ae: convolution neural network combined with autoencoder approach to detect survival chance of covid 19 patients. arXiv:2104.08954

Krizhevsky A, Sutskever I, Hinton GE (2017) Imagenet classification with deep convolutional neural networks. Commun ACM 60(6):84-90

Kumar R, Khan AA, Zhang S, Wang W, Abuidris Y, Amin W, Kumar J (2020) Blockchain-federated-learning and deep learning models for covid-19 detection using ct imaging. arXiv:2007.06537

Laguarta J, Hueto F, Subirana B (2020) COVID-19 artificial intelligence diagnosis using only cough recordings. IEEE Open J Eng Med Biol 1:275-281

Li L, Qin L, Xu Z, Yin Y, Wang X, Kong B, Bai J, Lu Y, Fang Z, Song Q et al (2020) Using artificial intelligence to detect COVID-19 and community-acquired pneumonia based on pulmonary CT: evaluation of the diagnostic accuracy. Radiology. 2020;296(2):E65-E71. https://doi.org/10.1148/radiol.2020200905 evaluation of the diag- nostic accuracy. Radiology. 2020;296(2):E65-E71. https://doi.org/ 10.1148/radiol.2020200905

Li Y, Xia L (2020) Coronavirus disease 2019 (covid-19): role of chest ct in diagnosis and management. Am J Roentgenol 214(6):1280 1286

Liu S, Georgescu B, Xu Z, Yoo Y, Chabin G, Chaganti S, Grbic S, Piat S, Teixeira B, Balachandran A, et al (2020) 3d tomographic pattern synthesis for enhancing the quantification of covid-19. arXiv:2005.01903

Liu X, Faes L, Kale AU, Wagner SK, Fu DJ, Bruynseels A, Mahendiran T, Moraes G, Shamdas M, Kern C et al (2019) A comparison of deep learning performance against health-care professionals in detecting diseases from medical imaging: a systematic review and meta-analysis. Lancet Digit Health 1(6):e271-e297

Loey M, Manogaran G, Khalifa NEM (2020) A deep transfer learning model with classical data augmentation and cgan to detect covid19 from chest ct radiography digital images. Neural Comput Appl pp 1-13

Loey M, Manogaran G, Taha MHN, Khalifa NEM (2021) A hybrid deep transfer learning model with machine learning methods for face mask detection in the era of the covid-19 pandemic. Measurement 167:108288

Loey M, Smarandache F (2020) Within the lack of chest Covid-19 Xray dataset: a novel detection model based on gan and deep transfer learning. Symmetry 12(4):651

Long C, Xu H, Shen Q, Zhang X, Fan B, Wang C, Zeng B, Li Z, Li $X$, Li H (2020) Diagnosis of the coronavirus disease (covid-19): rrt-pcr or ct? Eur J Radiol 126:108961

Luz E, Silva PL, Silva R, Moreira G (2020) Towards an efficient deep learning model for covid-19 patterns detection in $\mathrm{x}$-ray images. arXiv:2004.05717

Maghdid HS, Asaad AT, Ghafoor KZ, Sadiq AS, Khan MK (2020) Diagnosing covid-19 pneumonia from x-ray and ct images using deep learning and transfer learning algorithms. arXiv:2004.00038

Maghdid HS, Ghafoor KZ, Sadiq AS, Curran K, Rabie K (2020) A novel ai-enabled framework to diagnose coronavirus covid 19 using smartphone embedded sensors: design study. arXiv:2003.07434

Makris A, Kontopoulos I, Tserpes K (2020) Covid-19 detection from chest $\mathrm{x}$-ray images using deep learning and convolutional neural networks. In: 11th hellenic conference on artificial intelligence, pp 60-66

Minaee S, Kafieh R, Sonka M, Yazdani S, Soufi GJ (2020) Deep-covid: predicting covid-19 from chest $\mathrm{X}$-ray images using deep transfer learning. arXiv:2004.09363

Misra S, Jeon S, Lee S, Managuli R, Jang IS, Kim C (2020) Multichannel transfer learning of chest $\mathrm{x}$-ray images for screening of covid-19. Electronics 9(9):1388

Müller D, Rey IS, Kramer F (2020) Automated chest ct image segmentation of covid-19 lung infection based on 3d u-net. arXiv:2007.04774

Narin A, Kaya C, Pamuk Z (2020) Automatic detection of coronavirus disease (covid-19) using X-ray images and deep convolutional neural networks. arXiv:2003.10849

Nayak SR, Nayak DR, Sinha U, Arora V, Pachori RB (2020) Application of deep learning techniques for detection of covid-19 cases using chest X-ray images: a comprehensive study. Biomed Signal Process Control 64:102365

Nour M, Cömert Z, Polat K (2020) A novel medical diagnosis model for covid-19 infection detection based on deep features and Bayesian optimization. Appl Soft Comput $\mathrm{p} 106580$

Oh Y, Park S, Ye JC (2020) Deep learning covid-19 features on cxr using limited training data sets. IEEE Trans Med Imag 39:2688-2700

Pal A, Sankarasubbu M (2020) Pay attention to the cough: early diagnosis of covid-19 using interpretable symptoms embeddings with cough sound signal processing. arXiv:2010.02417 
Perumal V, Narayanan V, Rajasekar SJS (2020) Detection of covid19 using cxr and ct images using transfer learning and haralick features. Appl Intell 51:341-358

Piczak KJ (2015) Esc: dataset for environmental sound classification. In: Proceedings of the 23rd ACM international conference on Multimedia, pp. 1015-1018

Purswani JM, Dicker AP, Champ CE, Cantor M, Ohri N (2019) Big data from small devices: the future of smartphones in oncology. In: Seminars in radiation oncology, vol 29, pp 338-347. Elsevier

Quatieri TF, Talkar T, Palmer JS (2020) A framework for biomarkers of covid-19 based on coordination of speech-production subsystems. IEEE Open J Eng Med Biol 1:203-206

Rahaman MM, Li C, Yao Y, Kulwa F, Rahman MA, Wang Q, Qi S, Kong F, Zhu X, Zhao X (2020) Identification of covid-19 samples from chest $\mathrm{X}$-ray images using deep learning: a comparison of transfer learning approaches. J X-ray Sci Technol (Preprint) 1-19

Rahimzadeh M, Attar A, Sakhaei SM (2021) A fully automated deep learning-based network for detecting covid-19 from a new and large lung ct scan dataset. Biomed Signal Process Control 68:102588

Rahman T (2020) COVID-19 Radiography Database. "https://www. kaggle.com/tawsifurrahman/covid19-radiography-database/" Accessed 09 July 2021

Rezaei M, Azarmi M (2020) Deepsocial: social distancing monitoring and infection risk assessment in covid-19 pandemic. Appl Sci 10(21):7514

Rothe C, Schunk M, Sothmann P, Bretzel G, Froeschl G, Wallrauch C, Zimmer T, Thiel V, Janke C, Guggemos W et al (2020) Transmission of 2019-ncov infection from an asymptomatic contact in Germany. New Engl J Med 382(10):970-971

Schuller BW, Schuller DM, Qian K, Liu J, Zheng H, Li X (2020) Covid-19 and computer audition: an overview on what speech \& sound analysis could contribute in the sars-cov-2 corona crisis. arXiv:2003.11117

Sethy PK, Behera SK (2020) Detection of coronavirus disease (covid19) based on deep features. Preprints 2020030300:2020

Shalbaf A, Vafaeezadeh M et al (2020) Automated detection of covid19 using ensemble of transfer learning with deep convolutional neural network based on ct scans. Int J Comput Assist Radiol Surg $16: 115-123$

Sharifrazi D, Alizadehsani R, Roshanzamir M, Joloudari JH, Shoeibi A, Jafari M, Hussain S, Sani ZA, Hasanzadeh F, Khozeimeh F et al (2021) Fusion of convolution neural network, support vector machine and sobel filter for accurate detection of covid-19 patients using X-ray images. Biomed Signal Process Control 68:102622

Sharma N, Krishnan P, Kumar R, Ramoji S, Chetupalli SR, Ghosh PK, Ganapathy S, et al. (2020) Coswara-a database of breathing, cough, and voice sounds for covid-19 diagnosis. arXiv:2005.10548

Shih G, Wu CC, Halabi SS, Kohli MD, Prevedello LM, Cook TS, Sharma A, Amorosa JK, Arteaga V, Galperin-Aizenberg M et al (2019) Augmenting the national institutes of health chest radiograph dataset with expert annotations of possible pneumonia. Radiol Artif Intell 1(1):e180041

Shoeibi A, Khodatars M, Alizadehsani R, Ghassemi N, Jafari M, Moridian P, Khadem A, Sadeghi D, Hussain S, Zare A, et al. (2020) Automated detection and forecasting of covid-19 using deep learning techniques: a review. arXiv:2007.10785

Simonyan K, Zisserman A (2014) Very deep convolutional networks for large-scale image recognition. arXiv: 1409.1556

Sitaula C, Hossain MB (2020) Attention-based vgg-16 model for covid19 chest X-ray image classification. Appl Intell 51:2850-2863

Song Y, Zheng S, Li L, Zhang X, Zhang X, Huang Z, Chen J, Zhao H, Jie Y, Wang R, et al (2020) Deep learning enables accurate diagnosis of novel coronavirus (covid-19) with ct images. medRxiv
Stephen O, Sain M, Maduh UJ, Jeong DU (2019) An efficient deep learning approach to pneumonia classification in healthcare. $\mathrm{J}$ Healthcare Eng 2019

Ucar F, Korkmaz D (2020) Covidiagnosis-net: deep bayes-squeezenet based diagnostic of the coronavirus disease 2019 (covid-19) from X-ray images. Med Hypotheses 140:109761

Wang X, Peng Y, Lu L, Lu Z, Bagheri M, Summers RM (2017) ChestX-ray8: hospital-scale chest $\mathrm{X}$-ray database and benchmarks on weakly-supervised classification and localization of common thorax diseases. In: Proceedings of the IEEE conference on computer vision and pattern recognition, pp 2097-2106

Waheed A, Goyal M, Gupta D, Khanna A, Al-Turjman F, Pinheiro PR (2020) Covidgan: data augmentation using auxiliary classifier gan for improved covid-19 detection. IEEE Access 8:91916-91923

Wang J, Bao Y, Wen Y, Lu H, Luo H, Xiang Y, Li X, Liu C, Qian D (2020a) Prior-attention residual learning for more discriminative COVID-19 screening in CT images. IEEE Trans Med Imag 39(8):2572-2583

Wang L, Lin ZQ, Wong A (2020b) Covid-net: a tailored deep convolutional neural network design for detection of covid-19 cases from chest X-ray images. Sci Rep 10(1):1-12

Wang P, Zheng X, Ai G, Liu D, Zhu B (2020c) Time series prediction for the epidemic trends of covid-19 using the improved lstm deep learning method: case studies in russia, peru and iran. Chaos Solitons Fractals 140:110214

Wang S, Zha Y, Li W, Wu Q, Li X, Niu M, Wang M, Qiu X, Li H, $\mathrm{Yu} \mathrm{H}$ et al (2020d) A fully automatic deep learning system for COVID-19 diagnostic and prognostic analysis. Eur Respiratory J $56(2)$

Wang X, Deng X, Fu Q, Zhou Q, Feng J, Ma H, Liu W, Zheng C (2020e) A weakly-supervised framework for COVID-19 classification and lesion localization from chest CT. IEEE Trans Med Imag 39(8):2615-2625

Wu YH, Gao SH, Mei J, Xu J, Fan DP, Zhang RG, Cheng MM (2021) Jcs: an explainable covid-19 diagnosis system by joint classification and segmentation. IEEE Trans Image Process 30:3113-3126

Xie X, Zhong Z, Zhao W, Zheng C, Wang F, Liu J (2020) Chest ct for typical 2019-ncov pneumonia: relationship to negative rt-pcr testing. Radiology p. 200343

Xiong C, Hu S, Yang M, Luo W, Zhang L (2020) Mobile device data reveal the dynamics in a positive relationship between human mobility and covid-19 infections. Proc Natl Acad Sci 117(44):27087-27089

Xu X, Jiang X, Ma C, Du P, Li X, Lv S, Yu L, Ni Q, Chen Y, Su J et al (2020) A deep learning system to screen novel coronavirus disease 2019 pneumonia. Engineering

Yang K, Liu X, Yang Y, Liao X, Wang R, Zeng X, Wang Y, Zhang M, Zhang T (2020) End-to-end covid-19 screening with 3d deep learning on chest computed tomography

Yang X, He X, Zhao J, Zhang Y, Zhang S, Xie P (2020) Covid-ct-dataset: a ct scan dataset about covid-19. arXiv:2003.13865

Yoon SH, Lee KH, Kim JY, Lee YK, Ko H, Kim KH, Park CM, Kim YH (2020) Chest radiographic and ct findings of the 2019 novel coronavirus disease (covid-19): analysis of nine patients treated in korea. Korean J Radiol 21(4):494-500

Zhang H.t, Zhang Js, Zhang Hh, Nan Yd, Zhao Y, Fu Eq, Xie Yh, Liu W, Li Wp, Zhang Hj et al (2020) Automated detection and quantification of covid-19 pneumonia: $\mathrm{Ct}$ imaging analysis by a deep learning-based software. Eur J Nucl Med Mol Imag 47(11):25252532

Zhang J, Xie Y, Li Y, Shen C, Xia Y (2020) Covid-19 screening on chest $\mathrm{X}$-ray images using deep learning based anomaly detection. arXiv:2003.12338

Zhang J, Xie Y, Liao Z, Pang G, Verjans J, Li W, Sun Z, He J, Yi Li, CS (2020) Viral pneumonia screening on chest $X$-ray images using confidence-aware anomaly detection. arXiv:2003.12338 
Zhao J, Zhang Y, He X, Xie P (2020) Covid-ct-dataset: a ct scan dataset about covid-19. arXiv:2003.13865

Zhao JY, Yan JY, Qu JM (2020) Interpretations of "diagnosis and treatment protocol for novel coronavirus pneumonia (trial version 7)." Chin Med J 133(11):1347-1349

Zhou S, Zhu T, Wang Y, Xia L (2020) Imaging features and evolution on ct in 100 covid-19 pneumonia patients in Wuhan, China. Eur Radiol pp 1-9
Zulkifley MA, Abdani SR, Zulkifley NH (2020) Covid-19 screening using a lightweight convolutional neural network with generative adversarial network data augmentation. Symmetry 12(9):1530

Publisher's Note Springer Nature remains neutral with regard to jurisdictional claims in published maps and institutional affiliations. 